\title{
Reinfection cases by closely related SARS-CoV-2 lineages in Southern Brazil
}

\author{
Juliana Schons Gularte ${ }^{1}$ (D) Mariana Soares da Silva ${ }^{1} \cdot$ Meriane Demoliner $^{1} \cdot$ Alana Witt Hansen ${ }^{1}$.

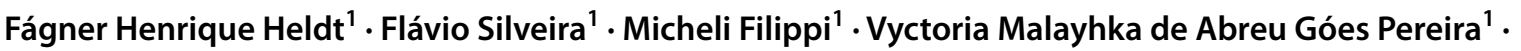 \\ Francini Pereira da Silva ${ }^{1}$ - Larissa Mallmann ${ }^{1}$. Pietra Fink ${ }^{1}$. Jéssica Luísa Laux ${ }^{1}$. Matheus Nunes Weber ${ }^{1}$. \\ Paula Rodrigues de Almeida ${ }^{1}$. Juliane Deise Fleck ${ }^{1} \cdot$ Fernando Rosado Spilki $^{1}$
}

Received: 23 July 2021 / Accepted: 19 September 2021 / Published online: 25 September 2021

(c) Sociedade Brasileira de Microbiologia 2021

\begin{abstract}
Severe acute respiratory syndrome coronavirus 2 (SARS-CoV-2) is responsible for the pandemic that started in late 2019 and still affects people's lives all over the world. Lack of protective immunity after primary infection has been involved with reported reinfection cases by SARS-CoV-2. In this study, we described two cases of reinfection caused by non-VOC (Variants of Concern) strains in southern Brazil, being one patient a healthcare worker. The four samples previously positive for SARS-CoV-2 by real-time quantitative reverse transcription polymerase chain reaction (RT-qPCR) were sequenced by a high-performance platform and the genomic analysis confirmed that lineages responsible for infections were B.1.91 and B.1.1.33 (patient 1), and B.1.1.33 and B.1.1.28 (patient 2). The interval between the two positive RT-qPCR for patients 1 and 2 was 45 and 61 days, respectively. This data shows that patients may be reinfected even by very closely related SARSCoV-2 lineages.
\end{abstract}

Keywords Reinfection $\cdot$ COVID-19 $\cdot$ B. $1 \cdot$ Healthcare worker $\cdot$ Brazil

\section{Introduction}

SARS-CoV-2 is described as a causative agent of a pneumonia disease that was firstly reported in late 2019 in Wuhan, Hubei province, central China [1]. The fast spread of the virus worldwide led World Health Organization (WHO) to characterize Coronavirus Disease (COVID-19) as a pandemic on March 11, 2020. Since then, there have been more than 210 million confirmed cases and more than 4 million deaths caused by COVID-19 globally (https://covid19.who. int/).

A suspected case of reinfection may be described when a person has symptoms compatible with COVID-19

Responsible Editor: Flavio Guimaraes Fonseca

Juliana Schons Gularte

julianaschons@feevale.br

1 Laboratório de Microbiologia Molecular, Universidade Feevale, Rodovia ERS-239, № 2755, Prédio Vermelho, Piso 1, sala 103, Vila Nova, Novo Hamburgo, Rio Grande do Sul CEP 93525-075, Brazil and a positive test result for SARS-CoV-2 following a period $\geq 45$ days from the first infection for which prolonged shedding of viral RNA and infection by another agent has been ruled out [2]. Furthermore, to confirm a reinfection case, SARS-CoV-2 complete genomic sequencing for both primary and secondary infections indicating that they belong to different genetic clades or lineages should be used to dismissing recurrence or prolonged viral excretion[2,3].

Reinfection cases might happen in convalescent or vaccinated individuals due to the lower neutralizing capacity of SARS-CoV-2 antibodies and partial immunity against new variants[4]. However, cases of reinfection have been reported for both VOC [5-7] and non-VOC strains [8-10]. In the State of Rio Grande do Sul (RS), South of Brazil, the lineages that were circulating during May to November of 2020 were, most of them, classified as B.1.1, B.1.1.33, B.1.1.248, and B.1.1.49[11, 12]. In late November, it was possible to see an increase of P.2 (Zeta) in the region[12]. One case of P.1 (Gamma) in late November was described in RS; however, only in late January of 2021 was evidenced the circulation of Gamma variant, coinciding with the peak of SARS-CoV-2 new cases, hospitalizations, and deaths[5]. 
Although many recent articles have highlighted the importance of possible Spike epitopes between former lineages and new variants as a potential cause of an increase in the frequency of reinfections[7, 13, 14], in this study, we reported two SARS-CoV-2 reinfection cases caused by nonvariant-related lineages in South Brazil. Both patients had a first RT-qPCR positive result in July of 2020 and a second in September of 2020, with 45 and 61 days between both infections for patients 1 and 2, respectively. Samples were sequenced and the lineage responsible for infections were B.1.91, B.1.1.33, and B.1.1.28.

\section{Material and methods}

\section{SARS-CoV-2 detection by RT-qPCR}

For SARS-CoV-2 detection, naso-oropharyngeal swab samples were received at Laboratório de Microbiologia Molecular of Universidade Feevale. Viral RNA was extracted with the commercial MagMAX ${ }^{\mathrm{TM}}$ CORE Nucleic Acid Purification Kit (Applied biosystems ${ }^{\mathrm{TM}}$, Thermo Fisher Scientific, Waltham, MA, USA) kit, using the automated equipment KingFisher ${ }^{\mathrm{TM}}$ Duo Prime (Thermo Fisher Scientific ${ }^{\mathrm{TM}}$ ). Real-time quantitative reverse transcription polymerase chain reaction (RT-qPCR) for SARS-CoV-2 selective for the envelope (E) gene was performed according to the Charite Institute, Berlin, Germany, protocols[15] and using AgPathID One-Step RT-PCR Reagents (Thermo Fisher Scientific $^{\mathrm{TM}}$ ). Assays were performed adding positive and negative controls. Both template control (NTC) and negative controls diluted in Milli-Q ultrapure RNase/DNase-free water were included to confirm lack of contamination among samples. The genome of the positive control used in all assays was deposited in the GISAID genomic database under access number EPI_ISL_2229839.

\section{SARS-CoV-2 genome sequencing and phylogenetic analysis}

Viral genome sequencing and phylogenetic analysis were performed as previously described by da Silva et al. (2021) [5]. Briefly, after naso-oropharyngeal swab samples extraction, as described above, reverse transcription reaction was carried out by SuperScript IV reverse transcriptase kit (Thermo Fisher Scientific, Waltham, MA, USA). Viral genome library preparation was carried out using the QIAseq SARS-CoV-2 Primer Panel paired for library enrichment and QIAseq FX DNA Library UDI Kit, according to the manufacturer's instructions (QIAGEN, Hilden, Germany). Illumina MiSeq platform (Foster City, CA, USA), using MiSeq Reagent Kit v3 (600-cycle), was used. After sequencing, Geneious Prime ${ }^{\mathrm{TM}}$ suite was used for genome assembly and editing. Briefly, FASTQ reads were imported, trimmed to remove low-quality sequences and primers used for library generation (BBDuk 37.25), and mapped against the reference sequence hCoV-19/Wuhan/WIV04/2019 (EPI_ISL_402124) available in the EpiCoV database from GISAID[16]. Sequences generated in this study were aligned with 97 Brazilian SARS-CoV-2 complete genomes and the reference sequence $(>29 \mathrm{~kb}$ ) that were retrieved from the GISAID database. Alignment using the reference sequence from Wuhan as the outgroup was performed using the Clustal Omega software. The Maximum likelihood phylogenetic analysis under the general time reversible model, allowing for a proportion of invariable sites and substitution rates, was inferred empirically in IQ-TREE v2.1.2 web server[17], especially for maximum-likelihood (ML, applying 200 replicates and 1000 bootstraps.

\section{Results}

Patient 1 is a 47-year-old woman living in Igrejinha city in the southernmost state of Brazil. In the 1st infection episode (hCoV-19/Brazil/LMM16242/2020, GISAID access: EPI_ ISL_2928341), she had symptoms that started on July 15, 2020, and naso-oropharyngeal swab samples collection for COVID-19 diagnostic by RT-qPCR was on July 20, 2020. In the reinfection (hCoV-19/Brazil/LMM25714/2020, GISAID access: EPI_ISL_2928147), the first symptoms appeared on August 31, 2020, and the sample was collected on September 3, 2020. The interval between the two positive RT-qPCR for SARS-CoV-2 was 45 days, and results were 20.15 and 16.77 CT (cycle threshold) values for $\mathrm{E}$ gene, for infection and reinfection, respectively. She was symptomatic in both events, being that, in the 1st episode, we did not have access to the full record of symptoms, and in the reinfection, she reported myalgia, dry cough, loss of appetite, and dyspnea.

Patient 2 is a 28-year-old woman living in São Leopoldo and working in Novo Hamburgo as a nursing technique, RS, Brazil. In the 1st infection (hCoV-19/Brazil/ LMM14996/2020, GISAID access: EPI_ISL_2928141), she had symptoms that started on July 10, 2020, and nasooropharyngeal swab sample collection for COVID-19 diagnostic was on July 13, 2020. In reinfection (hCoV-19/Brazil/LMM27281/2020, GISAID access: EPI_ISL_2928344), the first symptoms appeared on September 4, 2020, and the sample was collected on September 12, 2020. The result of RT-qPCR was 14.91 and 15.43 CT values for E gene, for infection and reinfection, respectively. The interval between the two positive results for SARS-CoV-2 was 61 days, and she was symptomatic in both events. In the 1 st episode of COVID-19, she reported experiencing flu symptoms, and, in the reinfection, she described body pain, tiredness, sore 
throat, headache, and runny nose. The patient's data were described in Table 1.

Whole-genome sequences for SARS-CoV-2 were covered in high quality for analyzed samples being two distinct lineages present in samples from the same person in different infections episodes demonstrating reinfection for both patients. According to Pangolin COVID-19 Lineage Assigner tool[18], sequences of patient 1 were classified as B.1.91 (hCoV-19/Brazil/LMM16242/2020) in infection and B.1.1.33 (hCoV-19/Brazil/LMM25714/2020) in reinfection (GISAID access: EPI_ISL_2928341 and EPI_ ISL_2928147, respectively). Sequences of patient 2 were classified as B.1.1.33 (hCoV-19/Brazil/LMM14996/2020) in infection and B.1.1.28 (hCoV-19/Brazil/LMM27281/2020) in reinfection (GISAID access: EPI_ISL_2928141 and EPI_ISL_2928344, respectively). Our phylogenetic analysis also confirmed these results clustering the four samples with their respective branches of B.1.91, B.1.1.28, and B.1.1.33 (Fig. 1).

\section{Discussion}

This study reported two reinfection cases of SARS-CoV-2 by non-VOC strains in the South of Brazil. Although reinfection cases of SARS-CoV-2 by VOC strains[5-7] have been linked to lower neutralizing capacity antibodies and partial immunity against new variants[4], a limited and transitory immunity induced by primary infection might be responsible for the occurrence of secondary one reflecting the virus ability to evade the previous immune responses[7] also in reinfection cases caused by non-VOC strains, as were described in others studies[8-10].

In our study, both patients had mild to moderate symptomatic cases in primary and secondary infection. Even though there are cases of reinfection with an increase in severity [5, 6], more cases have been showing less severity, suggesting partial immune protection from disease[19]. The four samples presented $\mathrm{CT}$ values for the $\mathrm{E}$ gene between 14 and 20. As already described, when CT values are higher than 24, the SARS-CoV-2 infectivity might be significantly reduced[20]. Therefore, reinfection cases described in our study contributed to onward transmission of the virus if isolation measures were not correctly applied.

There is an additional concern when a case of reinfection is linked to a health professional, and it is the history of patient 2 . This is due to the fact that transmission within the hospital environment can affect, in addition to professionals, patients who are already debilitated. Therefore, it is important to efficiently detect and manage healthcare-associated infections preventing transmission and complications[21].

Genomic surveillance of SARS-CoV-2 is responsible for reporting crucial issues to support epidemiological investigations such as the circulating lineages, the introduction of new sources of virus from other geographical areas, and the evolution of the virus in response to applied control measures[21]. The three lineages sequenced (B.1.91, B.1.1.28, and B.1.1.33) in our samples were descendants of B.1 lineage. Besides reinfection cases described above, SARS-CoV-2 lineage $B$ has been related with most Brazilian sequences[22], and also with coinfection cases that might be another relevant factor to the complex interaction between immune response systems and virus mutations[12].

Moreover, B.1.1.28 lineage described herein is related as an ancestral of the Variants of Interest (VOI) P.2 (Zeta) (alias for B.1.1.28.2)[23] and P.1 (Gamma) (alias for B.1.1.28.1) [24]. Another relevant issue is that B.1.1.28 has also been related to the emergence of putative novel SARS-CoV-2 variants circulating in Southern Brazil[12, 25]. Since this lineage originated VOIs, VOCs, and proposed new variants, it is a need to continually evaluate and track its circulation in the population. Besides genomic surveillance of SARS-CoV-2,

Table 1 Timeline of SARS-CoV-2 reinfection cases in two patients from Southern Brazil

\begin{tabular}{lllll}
\hline Patient & 1 & & 2 & \\
\hline Age & 47 & & 28 & Female \\
Gender & Female & & Infection & Reinfection \\
Infections cases & Infection & Reinfection & LMM 14996 & LMM 27281 \\
ID lab & LMM16242 & LMM25714 & July 10, 2020 & September 4, 2020 \\
Date of first symptoms & July 15, 2020 & August 31, 2020 & July 13, 2020 & September 12, 2020 \\
Sample collection & July 20, 2020 & September 3, 2020 & 14.91 & 15.43 \\
Ct value $(E)$ & 20.15 & 16.77 & B.1.1.33 & B.1.1.28 \\
Lineage & B.1.91 & B.1.1.33 & Symptomatic & Symptomatic \\
Clinical status & Symptomatic & Symptomatic & Flu symptoms & Body pain, tiredness, sore \\
Symptoms & No registry & Myalgia, dry cough, loss of & & throat, headache, and runny \\
& & appetite, and dyspnea & & nose
\end{tabular}




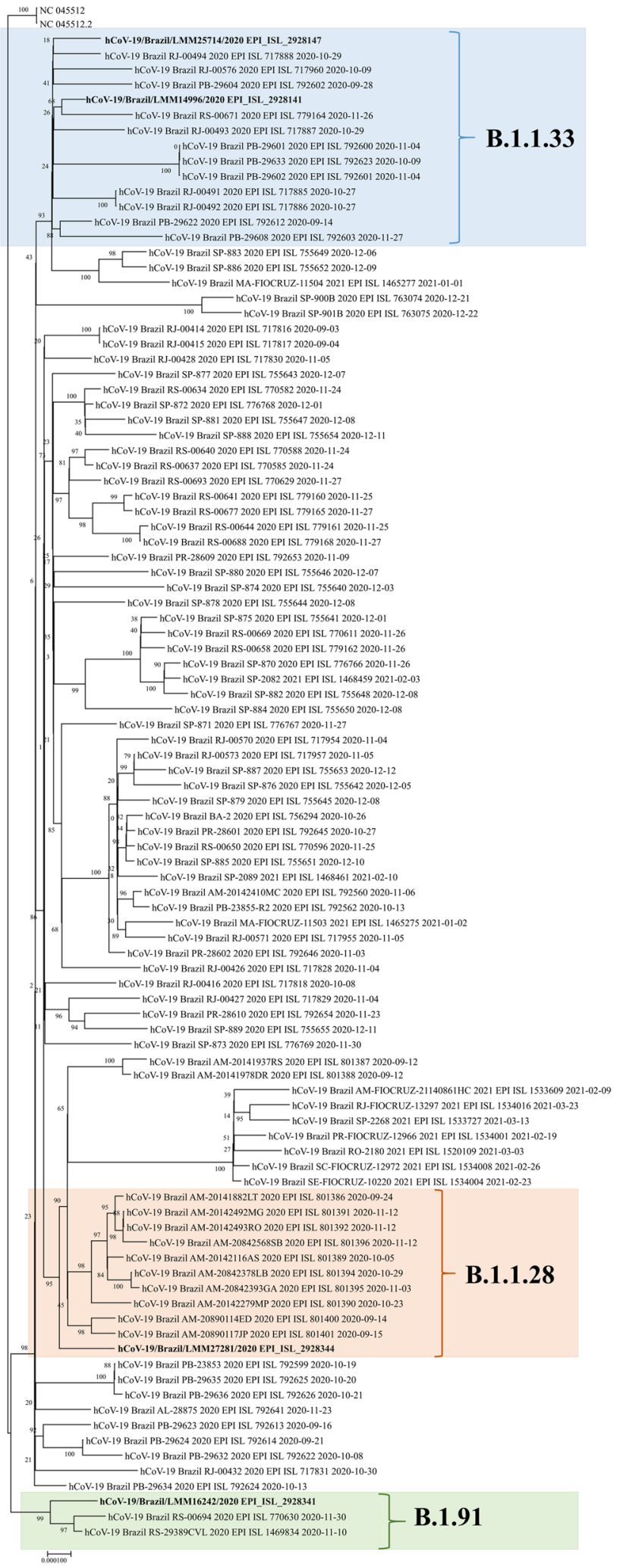

Fig. 1 Phylogenetic tree of SARS-CoV-2 complete genomes with the two cases of reinfection there is an evident need for clinical and epidemiological studies to enlighten how previous exposure through natural infection and vaccination protects against infection and reinfection with existing and newly emerging variants[4].

In accordance with our findings, we highlight the importance of continuous genomic monitoring of SARS-CoV-2 in samples from patients suspected of reinfection, especially because reinfection cases are not just linked to VOC that might have a significant reduction in virus neutralization by antibodies generated during infection or vaccination $[4,26]$. Therefore, the correct surveillance of these cases can help to elucidate the durability of the immune response against existing and new variants and even the need for vaccine boosters in the population. Also, it may assist in decisions planning the next measures that must be applied to respond to the pandemic, evaluating the specifics and necessities of each country or locality.

Supplementary Information The online version contains supplementary material available at https://doi.org/10.1007/s42770-021-00621-0.

Acknowledgements We thank the Brazilian Coordination for the Improvement of Higher-Level Personnel (CAPES), Foundation for Research Support of the State of Rio Grande do Sul (FAPERGS), and Brazilian National Council for Scientific Development (CNPq) for scholarships. See Supplementary Acknowledgments.

Author contribution Conceptualization, JSG and FRS; Formal analysis and investigation, JSG, MSS, MD, AWH, FHH, FS, MF, VMAGP, FPS, LM, PF, JLL, MNW, and PRA; Writing — original draft preparation, JSG; Writing — review and editing, MSS, JDF, and FRS; Funding acquisition, JDF and FRS; Resources, MNW, JDF, and FRS; Supervision, JDF and FRS.

Funding This work is an initiative of Rede Corona-ômica BR MCTI/ FINEP affiliated to RedeVirus/MCTI (FINEP=01.20.0029.000462/20, $\mathrm{CNPq}=404096 / 2020-4$ ), and FAPERGS (grant 21/2551-0000081-3).

Data availability Not applicable.

Code availability Not applicable.

\section{Declarations}

Ethics approval Project approved by the Research Ethics Committee (CEP) at Feevale University. Process number: CAAE: 33202820.7.1001.5348.

Consent to participate Not applicable.

Consent for publication Not applicable.

Conflict of interest The authors declare no competing interests. 


\section{References}

1. Zhou P, Lou YX, Wang XG et al (2020) A pneumonia outbreak associated with a new coronavirus of probable bat origin. Nature 579(7798):270-273. https://doi.org/10.1038/s41586-020-2012-7

2. Pan American Health Organization, World Health Organization. Interim guidelines for detecting cases of reinfection by SARSCoV-2. PAHO/WHO. Published 2020. https://www.paho.org/en/ documents/interim-guidelines-detecting-cases-reinfection-sarscov-2. Accessed 18 June 2021

3. European Centre for Disease Prevention and Control. Reinfection with SARS-CoV: considerations for public health response. ECDC. Published 2020. https://www.ecdc.europa.eu/en/publicatio ns-data/threat-assessment-brief-reinfection-sars-cov-2. Accessed 10 July 2021

4. Souza WM, Amorim MR, Sesti-Costa R, et al (2021) Neutralisation of SARS-CoV-2 lineage P.1 by antibodies elicited through natural SARS-CoV-2 infection or vaccination with an inactivated SARS-CoV-2 vaccine: an immunological study. Lancet Microbe. Published online July:1-9. https://doi.org/10.1016/S26665247(21)00129-4

5. da Silva MS, Demoliner M, Hansen AW et al (2021) Early detection of SARS-CoV-2 P.1 variant in Southern Brazil and reinfection of the same patient by P.2. Rev Inst Med Trop Sao Paulo 63:1-8. https://doi.org/10.1590/s1678-9946202163058

6. Harrington D, Kele B, Pereira S, et al (2021) Confirmed reinfection with severe acute respiratory syndrome coronavirus 2 (SARS-CoV-2) variant VOC-202012/01. Clin Infect Dis. Published online. https://doi.org/10.1093/cid/ciab014

7. Naveca F, Costa C, Nascimento V, et al. SARS-CoV-2 reinfection by the new Variant of Concern (VOC) P.1 in Amazonas,. Virological. Published online 2021:1-9. https://virological.org/t/ sars-cov-2-reinfection-by-the-new-variant-of-concern-voc-p-1-inamazonas-brazil/596

8. Amorim MR, Souza WM, Barros ACG et al (2021) Respiratory viral shedding in healthcare workers reinfected with SARSCoV-2, Brazil, 2020. Emerg Infect Dis 27(6):1737-1740. https:// doi.org/10.3201/eid2706.210558

9. Kulkarni O, Narreddy S, Zaveri L, Kalal IG, Tallapaka KB, Sowpati DT (2021) Evidence of severe acute respiratory syndrome coronavirus 2 (SARS-CoV-2) reinfection without mutations in the spike protein. Clin Infect Dis. Published online. https://doi. org/10.1093/cid/ciab136

10. Tillett RL, Sevinsky JR, Hartley PD et al (2021) Genomic evidence for reinfection with SARS-CoV-2: a case study. Lancet Infect Dis 21(1):52-58. https://doi.org/10.1016/S1473-3099(20) 30764-7

11. Franceschi VB, Caldana GD, de Menezes MA et al (2021) Genomic epidemiology of SARS-CoV-2 in Esteio, Rio Grande do Sul Brazil. BMC Genomics 22(1):1-17. https://doi.org/10.1186/ s12864-021-07708-w

12. da Silva Francisco R Jr, Benites LF, Lamarca AP et al (2021) Pervasive transmission of E484K and emergence of VUI-NP13L with evidence of SARS-CoV-2 co-infection events by two different lineages in Rio Grande do Sul, Brazil. Virus Res 296:198345. https://doi.org/10.1016/j.virusres.2021.198345

13. Resende PC, Bezerra JF, Teixeira de Vasconcelos RH, et al. Spike E484K mutation in the first SARS-CoV-2 reinfection case confirmed in Brazil, 2020. Virological. Published online 2021:1-8. https://virological.org/t/spike-e484k-mutation-in-the-first-sarscov-2-reinfection-case-confirmed-in-brazil-2020/584

14. Nonaka CKV, Franco MM, Gräf T et al (2021) Genomic evidence of SARS-CoV-2 reinfection involving E484K spike mutation Brazil. Emerg Infect Dis 27(5):1522-1524. https://doi.org/10.3201/ eid2705.210191

15. Corman V, Bleicker T, Brünink S, et al (2020) Diagnostic detection of 2019-NCoV by real-time RT-RCR. https://virologie-ccm. charite.de/en/

16. Shu Y, McCauley J (2017) GISAID: global initiative on sharing all influenza data - from vision to reality. Eurosurveillance 22(13):2-4. https://doi.org/10.2807/1560-7917.ES.2017.22.13. 30494

17. Nguyen LT, Schmidt HA, Von Haeseler A, Minh BQ (2015) IQTREE: a fast and effective stochastic algorithm for estimating maximum-likelihood phylogenies. Mol Biol Evol 32(1):268-274. https://doi.org/10.1093/molbev/msu300

18. Rambaut A, Holmes EC, O'Toole Á et al (2020) A dynamic nomenclature proposal for SARS-CoV-2 lineages to assist genomic epidemiology. Nat Microbiol 5(11):1403-1407. https:// doi.org/10.1038/s41564-020-0770-5

19. Babiker A, Marvil CE, Waggoner JJ, Collins MH, Piantadosia A (2021) The importance and challenges of identifying SARSCoV-2 reinfections. J Clin Microbiol 59(4):e02769-20. https:// doi.org/10.1128/JCM.02769-20

20. Bullard J, Dust K, Funk D et al (2020) Predicting infectious severe acute respiratory syndrome coronavirus 2 from diagnostic samples. Clin Infect Dis 71(10):2663-2666. https://doi.org/10.1093/ $\mathrm{cid} / \mathrm{ciaa} 638$

21. Meredith LW, Hamilton WL, Warne B et al (2020) Rapid implementation of SARS-CoV-2 sequencing to investigate cases of health-care associated COVID-19: a prospective genomic surveillance study. Lancet Infect Dis 20(11):1263-1272. https://doi.org/ 10.1016/S1473-3099(20)30562-4

22. Candido DS, Claro IM, de Jesus JG et al (2020) Evolution and epidemic spread of SARS-CoV-2 in Brazil. Science (80-) 369(6508):1255-1260. https://doi.org/10.1126/SCIENCE.ABD21 61

23. Voloch CM, da Francisco Silva R, de Almeida LGP et al (2021) Genomic characterization of a novel SARS-CoV-2 lineage from Rio de Janeiro, Brazil. J Virol 95(10):e00119-21. https://doi.org/ 10.1128/jvi.00119-21

24. Fujino T, Nomoto H, Kutsuna $S$ et al (2021) Novel SARS-CoV-2 variant in travelers from Brazil to Japan. Emerg Infect Dis 27(4):1243-1245. https://doi.org/10.3201/eid2704.210138

25. Silva MS da, Gularte JS, Demoliner M, et al (2021) Emergence of a putative novel SARS-CoV-2 P.X lineage harboring N234P and E471Q spike protein mutations in Southern Brazil. Res Sq. Published online:1-8. https://doi.org/10.21203/rs.3.rs-689012/v1

26. Bian L, Gao F, Zhang J et al (2021) Effects of SARS-CoV-2 variants on vaccine efficacy and response strategies. Expert Rev Vaccines 20(4):365-374. https://doi.org/10.1080/14760584.2021. 1903879

Publisher's note Springer Nature remains neutral with regard to jurisdictional claims in published maps and institutional affiliations. 